\title{
Symbiotic Relationship with Gut Microbiomes and Parkinson Disease
}

Review Article

Opeyemi Oluwasanmi Adeloye ${ }^{1}$, Oyeneyin Babatunde David ${ }^{2} \&$ Samuel Olawuwo ${ }^{3}$

${ }^{1}$ Rosad Neurodegerative Research Institute Jos, University of Medical Science Teaching Hospital, Ondo.

${ }^{2}$ University of Medical Science Teaching Hospital, Ondo.

${ }^{3}$ University of Jos, Jos Nigeria.

Corresponding Author

Author Email:

Adeloyeopeyemi123@gmail.com

\section{INTRODUCTION}

Parkinson disease (PD) is a progressive chronic nervous system disease resulted from decrease in amount of dopamine production in substantia nigra.

Parkinson's disease is one of the
Abstract: Parkinson disease (PD) is a progressive chronic nervous system disease resulted from decrease in amount of dopamine production in substantia nigra. Studies shows that human being evolved a symbiotic relationship with their gut microbiome, a complex microbial community composed of bacteria, archaea, protists, and viruses. The enteric nervous system (ENS) is a gateway for the bidirectional communication between the brain and the gut, mostly through the vagus nerve (VN). Environmental exposure plays a vital role in both the composition and functionality of the gut microbiome and may contribute to susceptibility to neurodegenerative disorders, such as Parkinson's disease (PD). The neuropathological hallmark of PD is the widespread appearance of alpha-synuclein aggregates in both the central and peripheral nervous systems, including the ENS. Studies suggest that gut toxins can induce the formation of a-syn aggregates in the ENS, which may then be transmitted in a prion-like manner to the CNS through the vagus nerve. PD is strongly associated with aging and its negative effects on homeostatic mechanisms protecting from inflammation, oxidative stress, and protein malfunction. In this mini-review we attempt to concisely summarize the papers in the field of the gut-brain axis and Parkinson's disease. We focus on evidence showing gut-association and related microbial-derived components of PD. Therefore, the gut microbiome emerges as a potential target for protective measures aiming to prevent PD onset.

Keywords: Parkinson's disease, enteric nervous system, dopamine, microbiomes.

most common neurodegenerative disorders. PD commoner in age categories over 55 years above, the risk related more with increase in age. Symptoms PD characterized primarily by rigidity, tremor, bradykinesia, speech changes, writing changes and postural instability. PD typically associated with the progressive loss of dopaminergic neurons located in the midbrain nucleus substantia nigra pars compacta ( $\mathrm{SNpc}$ ). Research revealed Symptoms of PD are motor impairments attributed to the depletion of the neurotransmitter dopamine in the striatum, a major target of the SNpc. The neuropathological hallmark of PD is the presence of Lewy bodies (LB), in SNpc neurons. LBs are composed mostly of $\alpha$-synuclein ( $\alpha$-syn) aggregates, whose aberrant soluble oligomeric conformations are thought to mediate its toxic effects. Alpha-syn is an intrinsically disordered protein (IDP), which lacks a stable 3D structure under physiological conditions and is characterized by exacerbated structural plasticity and conformational adaptability. As other IDPs possessing amyloidogenic regions, $\alpha$-syn can turn into a promiscuous binder leading to abnormal interactions and the development of PD. Studies provided a detailed 3D structure of functional $\alpha$-syn fibrils, using solid-state NMR spectroscopy. The study may serve as the basis for a better understanding of molecular mechanisms involved in $\alpha$-syn fibril nucleation and propagation. In addition, such structural information may provide useful insights on possible interactions of $\alpha$-synuclein with other proteins and small molecules and allow the emergence of new tools with potential to facilitate both the diagnosis and treatment of PD (e.g., imaging agents and therapeutic drugs). 


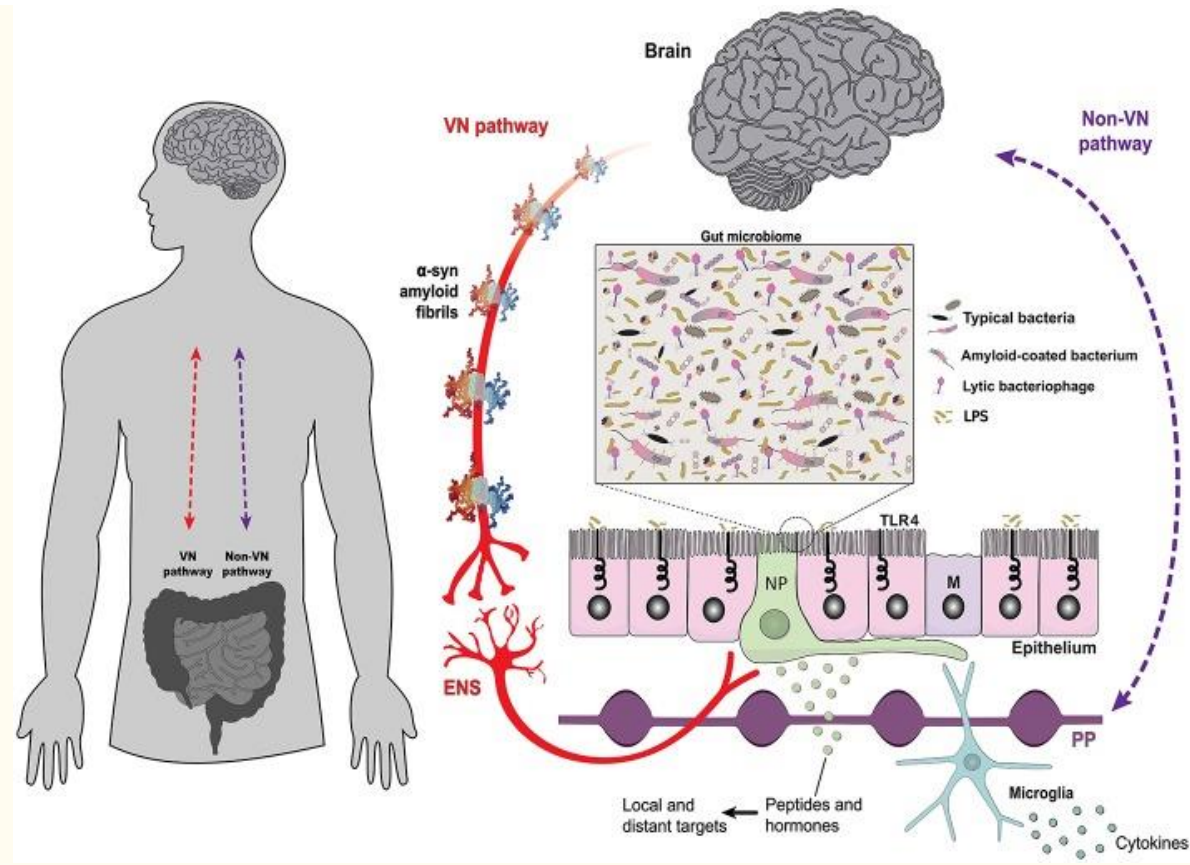

Figure 1

The gut epithelium is a multifunctional interface. The bidirectional interplay between the brain and the gut is mediated by neural, such as the vagus nerve, and humoral pathways, such as the lymphatic tissue and the bloodstream. A monolayer of epithelial cells separates the intestinal lumen and the complex gut microbiome from the underlying lymphoid and enteric nervous tissues. The structure of alpha-synuclein amyloid fibrils is based on atomic-resolution molecular data from NGL Viewer. Microbial antigens can cross the gut epithelium through microfold cells, playing a central role in localized inflammatory responses. Toll-like receptors are microbe-sensing proteins, present in intestinal epithelial cells, mediating recognition of commensal bacteria from the harmful/inflammatory ones. ENS, enteric nervous system; M, microfold cells; NP, neuropods; PP, Peyer's patches; TLR4, Toll-like receptor 4; VN, vagus nerve [adapted from Bohórquez et al]

Braak et al stated that aggregates of $\alpha$-syn fibrils are also found in neural tissue located outside the central nervous system (CNS) of PD patients, in both the autonomic and enteric nervous system (ENS), an outcome which may be associated with the non-motor symptoms of the disease. These findings led to propose a staging system for the progression of the disease following a specific pattern of $\alpha$-syn aggregates spreading from peripheral toward more centralized locations in the brain. The triggering event would be the invasion of vulnerable neural structures such as the olfactory epithelium and the ENS, which interface directly with the external environment, by a neurotoxicant. While both structures possess immunological and physical barriers protecting them against environmental insults, these barriers steadily deteriorate with aging

Many studies have supported the claim that $\alpha$-syn aggregates propagate in a prion-like manner via microtubule-associated transport along axons. In summary, the prion hypothesis of PD proposes that amyloidogenic $\alpha$-syn would induce a conformational change in the endogenous protein through permissive templating, convert it into a likeness of itself and propagate retrogradely through the vagus nerve or the olfactory tract from the ENS or the olfactory bulb, respectively. Even though definitive proof for this prion hypothesis is still 
missing and there is the controversial possibility that intestinal $\alpha$-syn aggregates have a brain origin, it has been shown that vagotomy is associated with a decreased risk for PD in humans. Also, grafted neurons in PD patients develop $\alpha$-syn aggregate pathology and $\alpha$-syn from PD patients can cause nigrostriatal degeneration in mice and non-human primates. Remarkably, exogenous $\alpha$-syn fibrils, either PD patient-derived or produced in $E$. coli, were able to seed the formation of LB-like inclusions which spread from the GI tract to the brain through the vagus nerve in rats.

Prior to Braak's hypothesis, however, there was already strong evidence pointing to the role played by exogenous toxins in the etiology of sporadic PD. For instance, postencephalitic parkinsonism (von Economo's disease), which has an autoimmune basis caused by a viral illness, is associated with degeneration of the basal ganglia. Additionally, the discovery of parkinsonism induced by 1-methyl-4-phenyl-1,2,4,5-tetrahydropyridine (MPTP) through self-administration, in 1982 brought to light a new class of xenobiotic substances that may cause PD-like symptoms by environmental contact. MPTP is a lipophilic compound which readily passes into the brain where it is converted by monoamine oxidase B (MAO-B) to 1-methyl-4-phenylpyridinium (MPP+) which is taken up by dopaminergic cells and impairs mitochondria respiration by poisoning complex 1 . There are many heterocyclic molecules that structurally resemble MPTP and are found in the brain from both endogenous and exogenous sources, such as tetrahydroisoquinolines (TIQ) and $\beta$-carbolines ( $\beta$-C). For instance, a TIQ derivative, salsolinol, which is produced by enterobacteria and has been found in the urine of PD patients, may have a double-faced, dose-dependent effect on the nigrostriatal pathway as either a harmful or protective agent.

The evidence for the role played by toxins in inducing parkinsonism and the relative scarcity of familial cases (about 10\%) underscore the importance of environmental and lifestyle factors over genetic ones in the etiology of the disease. Some chronic diseases have been associated with a phenomenon called evolutionary mismatch when ancestral traits are no longer adaptive in modern contexts. For instance, $\alpha$-syn is involved with normal synaptic function by regulating, among other things, the size of presynaptic vesicles and the assembly of SNARE proteins involved with the docking of synaptic vesicles to presynaptic membranes. However, as old age became common in humans after the early upper Paleolithic, the steady increase in longevity seen in modern times may have had a collateral effect on the protein homeostasis (proteostasis) network, which coordinates protein synthesis, folding, trafficking, disaggregation, and degradation. The breakdown of proteostasis, which is a common feature of many neurodegenerative diseases, means that misfolded proteins may accumulate due to lack of clearance or failure to refold into their native structures. In the case of prion-like proteins, this may cause further protein misfolding (template effect) leading to protein aggregation and ultimately cell death.

\section{THE GUT BRAIN AXIS AND PARKINSON DISEASE}

The gut-brain axis is mediated by communication between the CNS and the ENS. Through the ENS, the gut microbiota influences the development and function of all divisions of the nervous system and this association was established very early during the evolution of multicellular organisms. The first nervous system appeared more than 500 million years ago before the divergence of cnidarians and bilaterians, the two metazoan sister groups. That primitive brain had a simple structure, organized as a diffuse nerve net which controlled a restricted set of basic 
behaviors and was the template for the subsequent evolution of the mammalian ENS, which retained many of its basic structural characteristics, such as a network of nervous ganglia distributed in the myenteric and submucous plexuses. Higher vertebrates went to evolve an additional set of neural structures in the central nervous system (CNS), tasked with the control of more sophisticated behaviors. However, the ENS and the CNS maintain intense crosstalk through reciprocal connections mediated by the $\mathrm{VN}$ and pelvic nerve in mammals. As the main substrate for this information exchange, the vagus nerve is an attractive target of neurostimulation therapies for the treatment of psychiatric and gastrointestinal disorders.

The GI tract harbors a complex microbial ecosystem, consisting of bacteria, archaea, protists, and eukaryotic and prokaryotic viruses, also known as bacteriophages. The human microbiome has coevolved with its host, which keeps a tight leash on the intrinsic competitive nature of the microorganisms that comprise the microbiome, through both the nervous and the immune systems. This arrangement maximizes the benefits the host gains from the symbiotic relationship, including protection against pathogens, improved nutrition, and mental health. A sub-type of intestinal epithelial cells called enteroendocrine cells, provide a signaling pathway through which the microbiome interacts with the CNS via the vagus nerve. Enteroendocrine cells have diverse phenotypes and express a variety of peptides/hormones that can act as signaling molecules on distinct targets, both local and distant, and some are chemoreceptors responding to a variety of luminal stimuli. As other intestinal epithelial cells, enteroendocrine cells express toll-like receptors, allowing them to detect bacterial products, and activate vagal afferents through basal processes called neuropods

\section{CONCLUSION}

Aging is the main risk factor for the development of PD and delaying the aging process is neuroprotective to PD in animal models. Aging is also associated with the accumulation of neuroinflammatory sequelae and the breakdown of homeostatic mechanisms that protect against protein misfolding, oxidative stress, decreased mitochondrial function, etc. The gut, as one of the main gateways to environmental exposure to the brain, may contribute to increasing the susceptibility to these factors. The microbiome has a protective effect mediating this exposure, and dysbiosis seems to be a pivotal risk factor for PD and other neurological disorders. Thus, the adoption of preventive measures to ensure a healthy microbiome throughout the lifetime can potentially decrease the risk of developing PD and other neurodegenerative diseases. The widespread use of antibiotics, for instance, which can kill gut bacteria indiscriminately, can cause a shift of the microbiome to an alternative stable state with unknown consequences in the long term

\section{REFERENCES}

Kaelberer MM, Buchanan KL, Klein ME, Barth BB, Montoya MM, Shen X, et al. . A gut-brain neural circuit for nutrient sensory transduction. Science. (2018)

Bohórquez DV, Shahid RA, Erdmann A, Kreger AM, Wang Y, Calakos N, et al. . Neuroepithelial circuit formed by innervation of sensory enteroendocrine cells. J Clin Invest. (2015)

Anselmi L, Bove C, Coleman FH, Le K, Subramanian MP, Venkiteswaran K, et al. . Ingestion of subthreshold doses of environmental toxins induces ascending Parkinsonism in the rat. NPJ Parkinson Dis. (2018) 4:30.

Hawkes CH, Del Tredici K, Braak H. Parkinson's disease: a dual-hit hypothesis. Neuropathol Appl Neurobiol. (2007) 33:599-614. 
Gershon MD, Bursztajn S. Properties of the enteric nervous system: limitation of access of intravascular macromolecules to the myenteric plexus and muscularis externa. J Comp Neurol. (1978) 180:467-88.

Mellert TK, Getchell ML, Sparks L, Getchell TV. Characterization of the immune barrier in human olfactory mucosa. Otolaryngol Head Neck Surg. (1992) 106:181-8.

Attems J, Walker L, Jellinger KA. Olfaction and aging: a mini-review. Gerontology. (2015) 61:485-90.

Bowman GL, Dayon L, Kirkland R, Wojcik J, Peyratout G, Severin IC, et al. . Blood-brain barrier breakdown, neuroinflammation, and cognitive decline in older adults. Alzheimer Dement. (2018) 14:1640-50.

Reeve A, Simcox E, Turnbull D. Ageing and Parkinson's disease: why is advancing age the biggest risk factor? Ageing Res Rev. (2014) 14:19-30.

Okuzumi A, Kurosawa M, Hatano T, Takanashi M, Nojiri S, Fukuhara T, et al. . Rapid dissemination of alpha-synuclein seeds through neural circuits in an in-vivo prion-like seeding experiment. Acta Neuropathol Commun. (2018) 6:96.

Steiner JA, Quansah E, Brundin P. The concept of alpha-synuclein as a prion-like protein: ten years after. Cell Tissue Res. (2018) 373:161-73.

Holmqvist S, Chutna O, Bousset L, Aldrin-Kirk P, Li W, Björklund T, et al. . Direct evidence of Parkinson pathology spread from the gastrointestinal tract to the brain in rats. Acta Neuropathol. (2014) 128:805-20.

Eisenberg D, Jucker M. The amyloid state of proteins in human diseases. Cell. (2012) 148:1188-203.

Stopschinski BE, Diamond MI. The prion model for progression and diversity of neurodegenerative diseases. Lancet Neurol. (2017) 16:323-32.

Lawson VA, Furness JB, Klemm HM, Pontell L, Chan E, Hill AF, et al. . The brain to gut pathway: a possible route of prion transmission. Gut. (2010) 59:1643-51.

Ulusoy A, Phillips RJ, Helwig M, Klinkenberg M, Powley TL, Di Monte DA. Brain-to-stomach transfer of

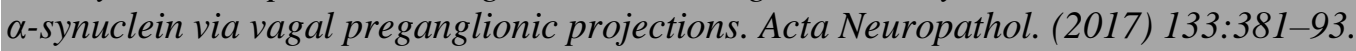

Liu B, Fang F, Pedersen NL, Tillander A, Ludvigsson JF, Ekbom A, et al. . Vagotomy and Parkinson disease: a Swedish register-based matched-cohort study. Neurology. (2017a) 88:1996-2002.

Svensson E, Horváth-Puhó E, Thomsen RW, Djurhuus JC, Pedersen L, Borghammer P, et al. . Vagotomy and subsequent risk of Parkinson's disease. Ann Neurol. (2015) 78:522-9.

Kordower JH, Chu Y, Hauser RA, Freeman TB, Olanow CW. Lewy body-like pathology in long-term embryonic nigral transplants in Parkinson's disease. Nat Med. (2008a) 14:504-6.

Kordower JH, Chu Y, Hauser RA, Olanow CW, Freeman TB. Transplanted dopaminergic neurons develop PD pathologic changes: a second case report. Mov Disord. (2008b) 23:2303-6.

Li J-Y, Englund E, Holton JL, Soulet D, Hagell P, Lees AJ, et al. . Lewy bodies in grafted neurons in subjects with Parkinson's disease suggest host-to-graft disease propagation. Nat Med. (2008) 14:501-3.

Dauer W, Przedborski S. Parkinson's disease: mechanisms and models. Neuron. (2003) 39:889-909.

Hughes AJ, Daniel SE, Kilford L, Lees AJ. Accuracy of clinical diagnosis of idiopathic Parkinson's disease: a clinico-pathological study of 100 cases. J Neurol Neurosurg Psychiatry. (1992) 55:181-4.

Garcia-Ruiz PJ, Chaudhuri KR, Martinez-Martin P. Non-motor symptoms of Parkinson's disease A review...from the past. J Neurol Sci. (2014) 338:30-3.

Braak H, Rüb U, Gai WP, Del Tredici K. Idiopathic Parkinson's disease: possible routes by which vulnerable neuronal types may be subject to neuroinvasion by an unknown pathogen. J Neural Transm. (2003) 110:517-36. 